Research Article

\title{
Signal Transmission Capability Improvement of a Telemetry Drill String Using the Reflected Signal at the Transmission Line Termination
}

\author{
Jia Jia, ${ }^{1}$ Yinao Su $\mathbb{D}^{1,2}$ Yue Shen, ${ }^{3}$ Gaixing Hu, ${ }^{4}$ Lingtan Zhang, ${ }^{3}$ and Limin Sheng ${ }^{2}$ \\ ${ }^{1}$ China University of Petroleum (East China), School of Petroleum Engineering, Qingdao 266580, China \\ ${ }^{2}$ CNPC Engineering Technology R\&D Company Limited, Beijing 102206, China \\ ${ }^{3}$ China University of Petroleum (East China), College of Science, Qingdao 266580, China \\ ${ }^{4}$ Oil and Gas Technology Institute, PetroChina Changqing Oilfield Company, Xi'an 710018, China
}

Correspondence should be addressed to Yinao Su; suyinao1949@163.com

Received 14 July 2020; Revised 26 September 2020; Accepted 23 October 2020; Published 18 November 2020

Academic Editor: Alessandro Lo Schiavo

Copyright $(2020$ Jia Jia et al. This is an open access article distributed under the Creative Commons Attribution License, which permits unrestricted use, distribution, and reproduction in any medium, provided the original work is properly cited.

\begin{abstract}
A telemetry drill string system consists of a string of wired drill pipes, and high-frequency signals pass the adjacent drill pipes through inductive couplers. Such a system is used to upload downhole information at high data transmission rates for measurements while drilling (MWD). Since the signal energy attenuates greatly in the system, many repeaters must be provided to ensure signal transmission. A reduction in the signal transmission in a telemetry drill string can extend the relay distance and improve the reliability of the transmission system. In this study, the transmission characteristic of the rod of the wired drill pipe is studied using transmission line theory, and the transmission characteristic of the inductive coupler is studied using high-frequency circuit theory. Using impedance matching between the transmission line of the rod and the inductive coupler, external impedance compensation elements, including a capacitor and a resistor, are recommended, and the electromagnetic parameters of the wired drill pipe are determined. Based on the determined electromagnetic parameters, certain changes in the external impedance compensation capacitance and resistance can lead to impedance mismatch between the transmission line and the inductive coupler. This will generate a reflected signal at the transmission line, and the vector superposes with the transmitted signal. The terminal reflection coefficient of the transmission line is controlled by increasing the compensation resistance value to enhance the signal amplitude, which can compensate the transmission loss when passing through the inductive coupler to a certain extent. Thus, the signal transmission capability of the telemetry drill string can be improved, allowing for long-distance signal transmission or drastically extending the relay distance, while maintaining a certain channel bandwidth.
\end{abstract}

\section{Introduction}

Measurement while drilling (MWD) is a modern drilling assistant technology for real-time downhole information measurement and transmission during the drilling process. MWD technology transmits drilling engineering parameters (e.g., drilling hydraulic pressure, wellbore temperature, inclination angle, azimuth angle, weight on bit, torque on bit, and bit vibration) and formation parameters (e.g., formation resistivity, rock porosity, and rock natural gamma ray) to the surface in real time [1-3]. The downhole information assists drilling engineers operate the rig correctly and drive the bit drill into oil and gas reservoir formations along corresponding tracks in order to drill quickly $[4,5]$, safely [6-8], and efficiently [9]. The downhole data transmission rates using wireless transmission technologies, including the mud pressure pulse $[10,11]$, the formation electromagnetic wave $[12,13]$, and the drill string sound wave [14-16], are very low (dozens of bit/s). In 2003, Grant Prideco developed the IntelliServ network system [17], a downhole data wired transmission system, using 
a drill pipe to transmit high-frequency electromagnetic signals, and its data transmission rate can reach $2 \mathrm{Mbit} / \mathrm{s}$ at a $2 \mathrm{MHz}$ channel bandwidth (using quadrature phase-shift keying modulation or QPSK modulation). In this system, the signal transmission requires a large number of repeaters, and the relay distance is the length of 30 drill pipes in a series. In laboratory experiments, $80 \mathrm{mV}$ signal amplitude can be obtained at the receiving end under a $6 \mathrm{~V}$ voltage source of excitation. This means that the voltage signal transmission coefficient within the relay distance is approximately 0.013 or $-18.8 \mathrm{db}$. In 2015 , the more reliable IntelliServ network system version 2 was developed. The reliability of the system was improved, and the maintenance cost was reduced in this version; however, the relay distance was still a 30-drill pipe length [18].

As Figure 1 shows, a telemetry drill string system consists of multiple wired drill pipes in series, and multiple repeaters are arranged to enable long-distance signal transmission. The length of the wired drill pipe is approximately $10.5 \mathrm{~m}$, including a rod (the length of the rod is approximately $10.36 \mathrm{~m}$ ) and a helical box joint at each end of the rod (approximately $0.14 \mathrm{~m}$ in length) [16]. The coaxial cable is embedded in the rod and terminates at single-turn inductive coils that are installed in annular magnetic grooves machined in the helical pin nose and helical box joint. The coaxial cable and coils form a closed loop to conduct the electric current. As Figure 2 shows, when the drill pipes are connected in series to form a drill string, two coils at the end of the adjacent wired drill pipe form an inductive coupler and transmit high-frequency voltage signals using electromagnetic induction.

Because the high-frequency signal is seriously attenuated when passing through the telemetry drill string channel, active repeaters must be located approximately every $315 \mathrm{~m}$ for signal reception, amplification, and forwarding [19]. Hence, more than 10 repeaters are needed for more than 3,000 m of conventional well depth, which increases the cost of the information telemetry system and reduces the reliability of signal transmission. Because the voltage transmission coefficient of the inductive coupler has a great influence on the transmission characteristics of the channel, current methods focus on reducing the signal energy loss of the inductive coupler to increase its voltage transmission coefficient in order to improve the signal transmission capability of the telemetry drill string. One such method involves reducing the eddy current loss by selecting the magnetic material of the inductive coupler and cutting the material into sections [20]. Another involves reducing the surface roughness of the ferrite material using a smooth processing treatment to narrow the internal spacing of the inductive couplers to reduce magnetic flux leakage [21]. Since the rod coaxial cable and the coupled coil form a series network, impedance matching between the coaxial cable and the inductor coupler at high frequency has a great influence on the signal transmission. However, none of the studies mentioned above considered this question, resulting in limited improvements to the signal transmission capability of the telemetry drill string channel.

The signal transmission ability of the telemetry drill string is reflected in the bandwidth of the channel and the voltage transmission coefficient of the channel. The broader the bandwidth, the higher the transmission rate of the signal. A higher voltage transmission coefficient shortens the relay distance and reduces the number of repeaters required in the channel. When the signal frequency is greater than $10 \mathrm{MHz}$ in the coaxial cables embedded in the rod of the wired drill pipes, the signal wavelength is close to the length of the rod, so the coaxial cable should be abstracted as a transmission line with distributed parameters, while the inductive coupler is the load of the transmission line. When multiple wired drill pipes are in series, the load of the drill pipe circuit is the equivalent input impedance of the next drill pipe. Because the electromagnetic parameters of the drill pipe have a great influence on signal transmission, how to determine the electromagnetic parameters of the wired drill pipe reasonably is the key to improving the channel transmission characteristics. Based on transmission line theory and circuit theory, this paper studies this problem and proposes a method to improve the channel transmission characteristics using an impedance matching analysis between the coaxial cable and inductive couplers.

\section{Channel Model and Analysis of the Channel Transmission Characteristics}

2.1. Determination of the Wired Drill Pipe Electromagnetic Parameters according to the Maximum Power Transmission of the Transmission Line. The circuit's structure and electromagnetic parameters influence its electrical signal transmission characteristics. The telemetry drill string channel is formed by connecting wired drill pipes in a series. The coaxial cable in the rod of the wired drill pipe was analyzed using the transmission line theory, and the inductive coupler is the load of the transmission line of the rod. According to transmission line theory, when the impedances of the transmission line and the inductive coupler match, the transmission line can realize the maximum power transmission. Thus, the determination of the electromagnetic parameters of the wired drill pipe using impedance matching between the transmission line and the inductive coupler can theoretically obtain the best transmission of signal power on the transmission line [22]. As shown in Figure 3, impedance matching can be realized using impedance compensation elements composed of a resistor and a capacitor in parallel at the connection of the end of the transmission line and the primary coil of the inductive coupler.

According to the circuit analysis, the mathematical model of the electromagnetic parameters of the transmission line and inductive coupler was established, and the electromagnetic parameters of the wired drill pipe were determined using mathematical calculation. The inductive coupler is symmetrical; $r_{m}$ is the internal resistance of the coil; $L$ is the coil inductance; $k$ is the coupling coefficient; the mutual inductance is $M=k L ; \widehat{R}$ is the resistance per unit length of the transmission line; $\widehat{G}$ is the conductivity per unit length of the transmission line; $\widehat{L}$ is the inductance per unit length of the transmission line; $\widehat{C}$ is the capacitance per unit length of the transmission line; $l$ is the length of the rod of the wired drill pipe; $f$ is the design frequency; $\omega=2 \pi f$ is the 


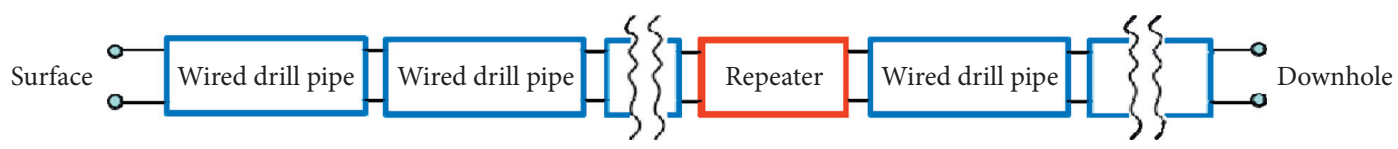

Figure 1: Telemetry drill string system.

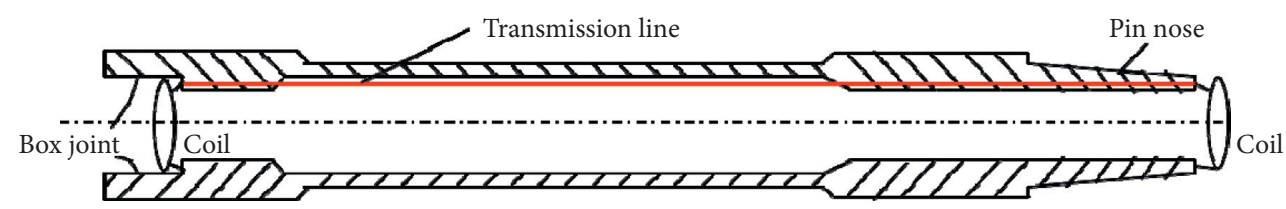

Figure 2: Cutaway of a wired drill pipe.

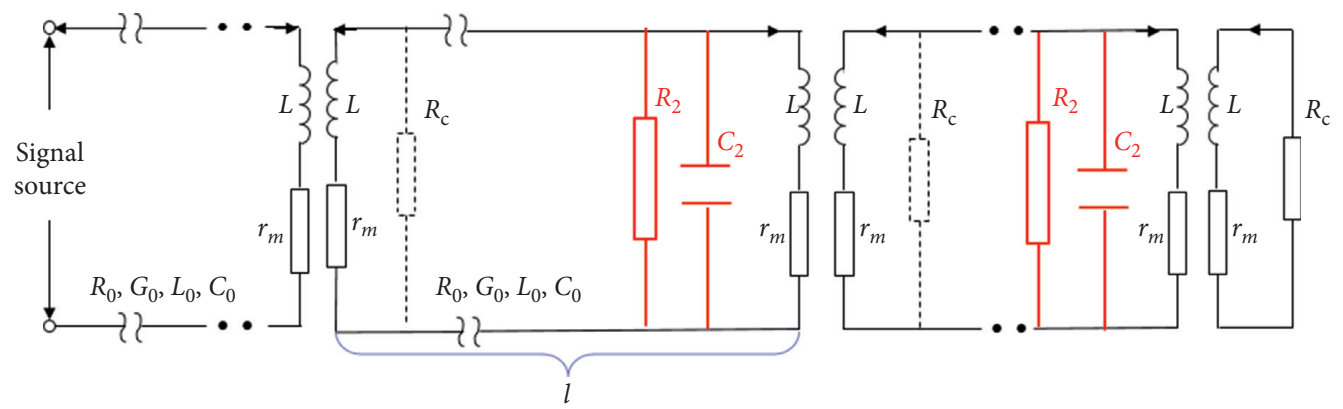

FIGURE 3: Impedance matching between the transmission line at the rod body and the inductive coupler.

angular frequency; $R_{c}=\sqrt{\widehat{L} / \widehat{C}}$ is the characteristic impedance of the transmission line under high-frequency conditions; and $R_{2}$ and $C_{2}$ are the resistance and capacitance of the impedance compensation elements, respectively. In order to realize the impedance matching of the transmission line and inductive coupler, the parallel impedance formed by the inductive coupler input impedance and the impedance compensation elements should be equal to the characteristic impedance of the transmission line. Thus,

$$
\frac{1}{R_{2}}=\frac{1}{j \omega_{d} C_{2}}+\frac{1}{R_{i n}+j X_{i n}}+\frac{1}{R_{c}},
$$

where $R_{\text {in }}=r_{m}+\left(\omega_{c}^{2} L^{2} k^{2}\left(R_{c}+r_{m}\right) /\left(R_{c}+r_{m}\right)^{2}+\left(\omega_{d} L\right)^{2}\right)$ and $X_{\text {in }}=\omega_{d} L-\left(\omega_{d}^{3} L^{3} k^{2} /\left(R_{c}+r_{m}\right)^{2}+\left(\omega_{d} L\right)^{2}\right)$.

The mathematical expressions of the resistance and capacitance of the impedance compensation elements can be obtained using equation (1):

$$
\left\{\begin{array}{l}
R_{2}=\frac{R_{c}\left(R_{\mathrm{in}}^{2}+X_{\mathrm{in}}^{2}\right)}{\left(R_{\mathrm{in}}^{2}+X_{\mathrm{in}}^{2}\right)-R_{\mathrm{in}} R_{c}}, \\
C_{2}=\frac{X_{\text {in }}}{\omega_{d}\left(R_{\mathrm{in}}^{2}+X_{\mathrm{in}}^{2}\right)} .
\end{array}\right.
$$

According to equation (2), when $R_{\text {in }}=R_{c} / 2, R_{2}$ reaches its maximum value and consumes the minimum loss of signal power. Therefore, the expression of the coil inductance of the coupler can be obtained as follows:

$$
L=\frac{R_{c}+r_{m}}{\omega_{d}} \sqrt{\frac{R_{c}-2 r_{m}}{2 k^{2}\left(R_{c}+r_{m}\right)-\left(R_{c}-2 r_{m}\right)}} .
$$

The constraint condition for the coupling coefficient of the inductive coupler is obtained using equation (3):

$$
\sqrt{\frac{R_{c}-2 r_{m}}{2\left(R_{c}+r_{m}\right)}}<k \leq 1 \text {. }
$$

As Figure 4 shows, the primary and secondary coils of the inductive coupler are single-turn circular coils placed in the annular high-frequency ferrite groove.

The relative permeability of ferrite is

$$
\mu_{r}=\frac{L}{4 \pi \times 10^{-7} d_{c} \int_{\left(d_{1} / 2\right)}^{\left(d_{2} / 2\right)} \int_{0}^{2 \pi}\left(\left(\sqrt{d_{c}^{2}+4 r^{2} \cos ^{2} \varphi-4 d_{c} r \cos \varphi}\right) /\left(\left(\sqrt{d_{c}^{2}+4 r^{2}-4 d_{c} r \cos \varphi}\right)^{3}\right)\right) r \mathrm{~d} r \mathrm{~d} \varphi},
$$




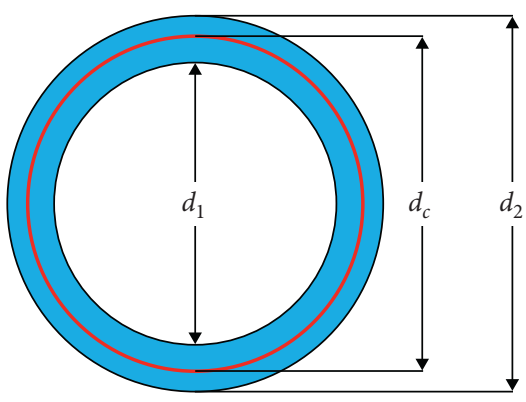

Ferrite

— Coil

FIgURE 4: Structure of primary and secondary side coils of the inductive coupler.

where $d_{\mathrm{c}}$ is the diameter of the coil, $d_{1}$ is the inner diameter of ferrite, and $d_{2}$ is the outer diameter of ferrite.

The resistance of the coil conductor is

$$
r_{\mathrm{m}}=\frac{4 d_{\mathrm{c}} \rho}{d_{0}^{2}},
$$

where $R_{0}$ is the diameter of the coil conductor and $\rho$ is the conductivity of the coil conductor.

To obtain the value of the determined electromagnetic parameters of the wired drill pipes, such as the characteristic impedance of the coaxial cable, the resistance of the coil conductor, the coil inductance, the coupling coefficient, the relative permeability of ferrite, and the resistance and capacitance values of the impedance compensation elements, the basic calculation parameters included the design frequency, capacitance per unit length of the transmission line, inductance per unit length of the transmission line, diameter of the coil, inner and outer diameters of ferrite, diameter of the coil conductor, and the conductivity of the coil conductor.

\subsection{Mathematical Model of the Channel Transfer Function.} The transmission characteristic of the channel reflects the transmission capability of all frequency components of the signal. The high-frequency electromagnetic signal transmitted using a telemetry drill string is a frequency band signal with a certain bandwidth. The signal spectrum is symmetrically distributed with the carrier frequency as the center. The signal bandwidth determines the transmission rate of information. Therefore, the frequency characteristics of the channel should meet the frequency distribution characteristics of the signal. The transmission function of the channel of the telemetry drill string is the product of the voltage transmission coefficients of the multiple wired drill pipes, which are related to the electromagnetic parameters of the channel, the frequency, and the number of wired drill pipes. The mathematical model of the channel of the voltage transmission function can be established through the circuit analysis of Figure 3.

The voltage transmission coefficient of the $i$-th coaxial cable can be established using transmission line theory:

$$
\eta_{d i}(\omega)=\left|\frac{1+\Gamma_{i}}{e^{\gamma l}+\Gamma_{i} e^{-\gamma l}}\right|,
$$

where $\Gamma_{i}=\left(Z_{L i}^{\prime}-R_{c}\right) /\left(Z_{L i}^{\prime}+R_{c}\right)$ is the reflection coefficient of the transmission line terminal; $Z_{L i}^{\prime}=\left(\left(1 / R_{2}\right)+j \omega C_{2}+\right.$ $\left.\left(1 / Z_{c i}\right)^{-1}\right)$ is the load impedance of the transmission line terminal; $Z_{c i}=r_{m}+j \omega L+\left(\omega^{2} k^{2} L^{2} / r_{m}+j \omega L n+q Z_{L i}\right)$ is the input impendence of the inductive coupler; $Z_{L(i+1)}=$ $R_{c}\left(e^{\gamma l}+\Gamma_{i} e^{-\gamma l} / e^{\gamma l}-\Gamma_{i} e^{-\gamma l}\right)$ is the load impedance of the secondary coil of the $i$-th inductive coupler; and $\gamma=\sqrt{(\hat{R}+\operatorname{tjn} \omega q \hat{L})(\hat{G}+\operatorname{tjn} \omega q \hat{C})}$ is the propagation constant of the transmission line.

Owing to the wide frequency band of the signal, a frequency component that does not meet the design frequency will generate a reflected signal at the terminal of the transmission line. The reflected signal's influence on the transfer function is expressed using a terminal reflection coefficient, which is defined as the vector ratio of the transmitted signal and reflected signal [23]. The reflection coefficient is a vector function; its module value is the ratio of the reflected signal amplitude to the transmitted signal amplitude, and its phase is the phase difference between the reflected signal and the transmitted signal.

The voltage transmission coefficient of the $i$-th inductive coupler is established on the basis of the high-frequency transformer circuit analysis:

$$
\eta_{c i}(\omega)=\left|\frac{j \omega M Z_{c i}}{\left(r_{m}+j \omega L+Z c i\right)\left(r_{m}+j \omega L\right)+\omega^{2} k^{2} L^{2}}\right| .
$$

The voltage transmission coefficient of the $i$-th wired drill pipe is the product of the transfer function between the transmission line and the inductive coupler:

$$
\eta_{i}(\omega)=\eta_{c i}(\omega) \eta_{d i}(\omega)
$$

The channel consists of $n$ wired drill pipes in series, and its voltage transmission coefficient can be expressed as

$$
\eta(\omega, n)=\prod_{i=1}^{n} \eta_{i}(\omega)=\left|\prod_{i=1}^{n}\left(\frac{j \omega M Z_{c i}}{\left(r_{m}+j \omega L+Z_{c i}\right)\left(r_{m}+j \omega L\right)+\omega^{2} k^{2} L^{2}} \frac{1+\Gamma_{i}}{e^{\gamma l}+\Gamma_{i} e^{-\gamma l}}\right)\right| .
$$

2.3. Transmission Characteristics' Improvement. For a channel of a certain length, its voltage transfer function can be analyzed as the change in the voltage transmission coefficient with frequency, the results of which are called the frequency domain characteristics of the channel or the transmission characteristics of the channel. Because the 
inductive coupler is a high-frequency transformer with a turn ratio of $1: 1$ and ferrite experiences magnetic field leakage and an eddy current loss under high frequencies, the voltage transmission coefficient of the inductive coupler is typically less than one. If the voltage transmission coefficient of the transmission line is one, the product of the voltage transmission coefficient of the wired drill pipe will sharply reduce the transmission capability of the channel. Owing to the limited improvement in the voltage transmission coefficient of the inductive coupler, a repeater was required to continue the signal transmission after a short distance of signal transmission. It can be seen from equation (10) that the terminal reflection coefficient of the transmission line will influence the frequency domain characteristics of the channel. When the signal is reflected, the voltage of the transmission line is the vector superposition of the transmitted signal voltage and the reflected signal voltage. According to the trigonometric calculation and analysis of the signal vector, if the reflection coefficient phase is less than $120^{\circ}$, the vector superposition of the reflected signal voltage and the transmitted signal voltage can enhance the signal amplitude of the transmission line, which can improve the voltage transmission coefficient of the transmission line. This will then compensate for the energy attenuation to a certain extent when the signal passes through the inductive coupler. When the voltage transmission coefficient of the wired drill pipe is close to one, the signal transmission characteristics of the channel are greatly improved. Similar methods have been applied in other fields [24].

In summary, the first step of the method was to determine the electromagnetic parameters of the wired drill pipe based on impedance matching between the transmission line and the inductive coupler. Proper changes in the capacitance and resistance of the impedance compensation elements will lead to an impedance mismatch between the transmission line and the inductive coupler, causing a reflected signal to be generated at the transmission line terminal. If the phase value between the reflected signal and the transmitted signal is less than $120^{\circ}$ in a certain frequency band, the channel will present a higher-voltage transmission coefficient accompanying a certain frequency bandwidth, thus realizing the long-distance transmission of high-frequency broadband voltage signals.

\section{Numerical Calculation and Simulation Analysis}

The calculation conditions were as follows: design frequency $f_{d}=10 \mathrm{MHz}$; length of the rod of the wired drill pipe $l=10.36 \mathrm{~m}$; resistance per unit length of the transmission line $\widehat{R}=0.0797 \Omega / \mathrm{m}$; capacitance per unit length of the transmission line $\widehat{C}=6.435 \times 10^{-11} \mathrm{~F} / \mathrm{m}$; inductance per unit length of the transmission line $\widehat{L}=3.798 \times 10^{-7} \mathrm{H} / \mathrm{m}$; conductivity per unit length of the transmission line $\widehat{G}=1.351 \times 10^{-16} \mathrm{~S} / \mathrm{m}$; and characteristic impedance of the transmission line $R_{c}=76.8 \Omega$. Then, according to equation (4), the coupling coefficient value was set at $k=0.8$, and according to equation (3), the coil inductance value was set at $L=2.3 \mu \mathrm{H}$, and the diameter of the coil conductor was $d_{0}=7.5 \times 10^{-4} \mathrm{~m}$. According to equation (2), the resistance value of the impedance matching compensation element was $R_{2}=137 \Omega$; the capacitance value of the impedance matching compensation element was $C_{2}=171 \mathrm{pF}$; the inner diameter of ferrite was $d_{1}=0.124 \mathrm{~m}$; the outer diameter of ferrite was $d_{2}=0.146 \mathrm{~m}$; the diameter of the coil was $d_{c}=0.135 \mathrm{~m}$; and the conductivity of the coil conductor was $\rho=1.75 \times 10^{-8} \Omega \mathrm{m}$. Finally, according to equation (6), the resistance value of the coil was $r_{m}=0.017 \Omega$, and according to equation (5), the relative permeability value of the ferrite material was $\mu_{r}=149$.

\subsection{Analysis of the Channel Transmission Characteristics}

3.1.1. Influence of the Compensation Capacitance on the Channel Transmission Characteristics. While maintaining the value of the compensation resistance at $R_{2}=137 \Omega$, the value of the compensation capacitance was changed from the impedance matching value to $C_{2}=80 \mathrm{pF}$ and $C_{2}=$ $350 \mathrm{pF}$.

According to equation (10), the voltage transmission coefficient of the channel consisting of multiple wired drill pipes under the influence of frequency was calculated. Figure 5 shows the frequency characteristic curve of the voltage transmission coefficient of the channel.

It can be seen from Figure 5 that changing the compensation capacitance caused a great change in the characteristics of the channel, but the passband of the channel was not obviously changed. Although the shape of the first passband in Figure 5(b) was better, the peak value of the transmission coefficient in the passband decreased sharply with an increase in the channel length, which is not suitable for long-distance signal transmission, indicating that changing the compensation capacitance will not produce better channel characteristics.

3.1.2. Influence of the Compensation Resistance on the Channel Transmission Characteristics. As the compensation resistance is connected in parallel at the end of the drill pipe transmission line, increasing the compensation resistance can not only reduce the energy loss of the voltage signal due to resistance but also change the impedance matching state of the transmission line. While maintaining the compensation capacitance at $C_{2}=171 \mathrm{pF}$, the value of the compensation resistance was changed from the impedance matching value to $R_{2}=500 \Omega$ and $R_{2}=1000 \Omega$. According to equation (10), the voltage transmission coefficient of the channel consisting of multiple wired drill pipes under the influence of frequency was calculated. Figure 6 shows the frequency characteristic curve of the voltage transmission coefficient of the channel.

Four observations can be made from Figure 6. First, there are two passbands in the transmission characteristic curve of the channel, and the bandwidths of these two passbands are basically the same, but the symmetry of the second passband is better than that of the first passband. This is much more suitable for a signal transmission channel. Second, although the peak value of the voltage transmission 


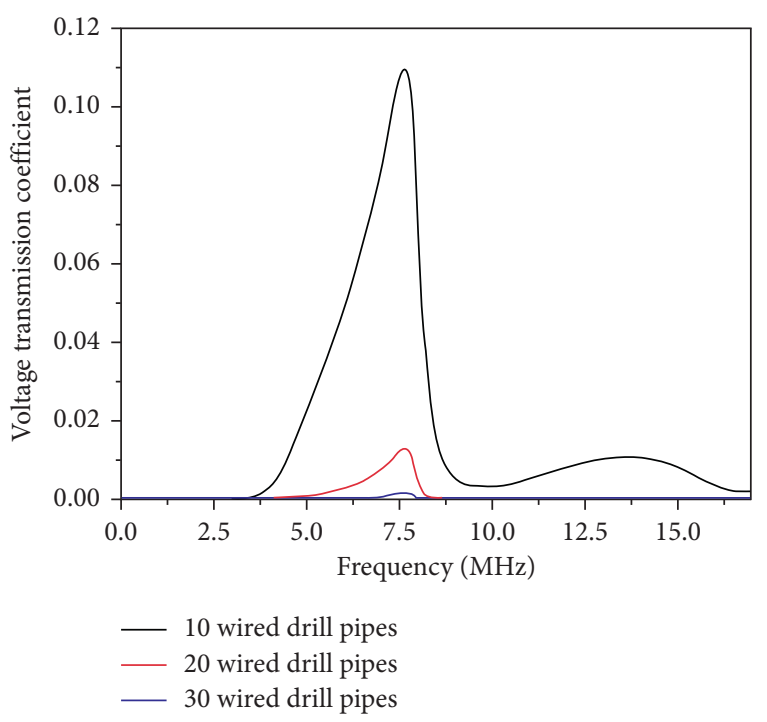

(a)

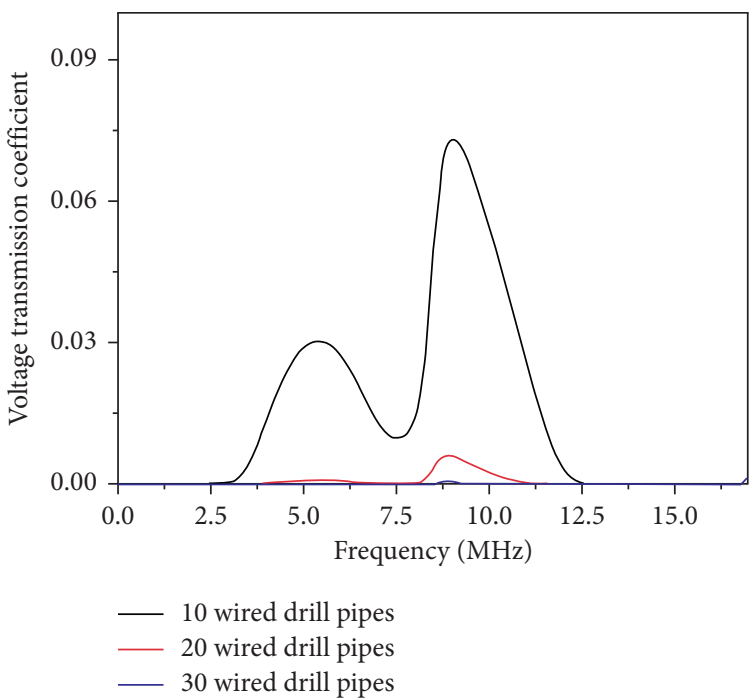

(b)

Figure 5: Influence of the compensation capacitance on the channel transmission characteristics. (a) $C_{2}=80 \mathrm{pF}$. (b) $C_{2}=350 \mathrm{pF}$.

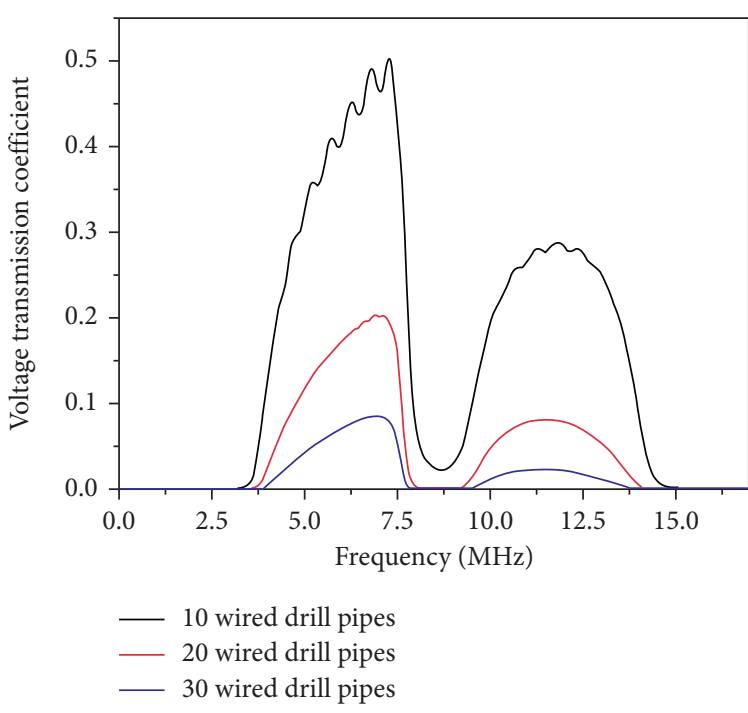

(a)

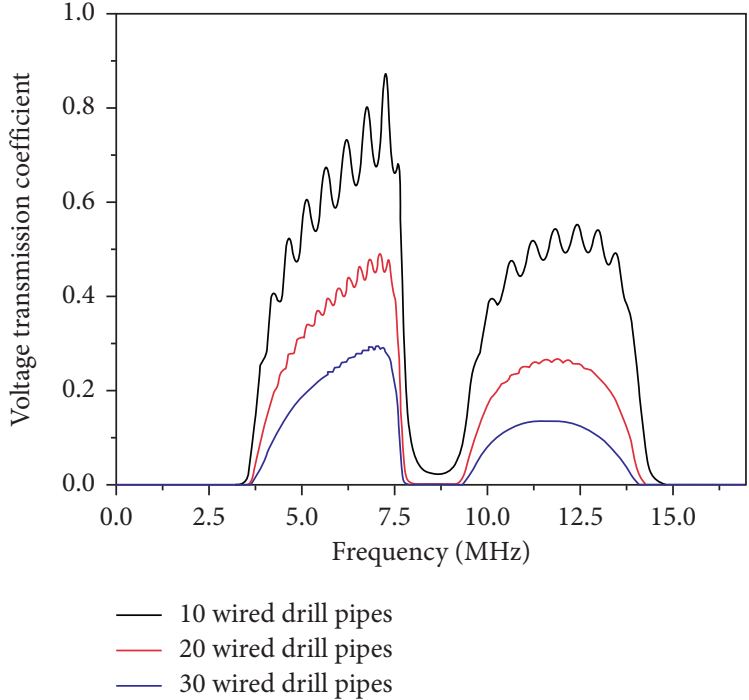

(b)

Figure 6: The influence of the compensation resistance on the channel transmission characteristics. (a) $R_{2}=500 \Omega$. (b) $R_{2}=1000 \Omega$.

coefficient in the passband obviously decreased with the length of the channel, the passband width was basically unchanged, which is conducive to the transmission of the frequency band signal. Third, when the channel is short, the voltage transmission coefficient will have a large, periodic zigzag fluctuation, which can be referred to as the distorted characteristics of the channel. This zigzag fluctuation is caused by the change in the reflection coefficient of each transmission line of the wired drill pipe with the frequency. This phenomenon of channel fluctuation can also be seen in the acoustic transmission condition in the MWD [25]. The voltage transmission coefficient of each transmission line is influenced by the frequency change to a different extent.
When the number of wired drill pipes is small, the difference of this influence is prominent, which results in great fluctuations of the frequency characteristic curve in the passband. When the number of wired drill pipes is large, the smoothing effect of the product of the voltage transmission coefficient of each drill pipe makes the channel frequency characteristic curve smooth. The large zigzag fluctuation of the voltage transmission coefficient in the passband will cause serious distortion of signal transmission. To solve this problem, when the number of wired drill pipes, $n$, is relatively small, because the signal amplitude transmitted through the channel is still large, the signal can pass through a filter whose transfer function is $H(\omega)=\eta(\omega, n)^{-1}$. The 
mathematical model of the voltage transfer function, $\eta(\omega, n)$, in the channel of the number of wired drill pipes, $n$, is used to compensate for the change in the signal frequency component caused by the characteristic distortion of the channel to theoretically eliminate the signal transmission distortion caused by the channel characteristic distortion. Fourth, when the number of drill pipes is the same, the peak value of the voltage transmission coefficient of the passband in Figure 6(b) is much higher than that of the corresponding passband in Figure 6(a), indicating that the larger deviation of the resistance value from the compensation resistance value generates higher reflection voltage at the terminal end of the transmission line. This leads to a better compensation result of the signal amplitude decrease when passing the inductive coupler. This means the transmission capability of the channel can be effectively improved by increasing the compensation resistance.

However, although the increase of $R_{2}$ can improve the voltage transmission coefficient in the passband to a certain extent, $R_{2}$ should be capped. The numerical calculation results showed that when under the same number of drill pipes in series, compared with the channel frequency characteristics when $R_{2}=1000 \Omega$, if $R_{2}=1000 \Omega$, with a gradual increase in $R_{2}$, the signal reflection at each rod transmission line terminal in the channel gradually became larger. In addition, the voltage transmission coefficient in the two passbands of the channel all increased, but the increasing trend gradually declined, and the bandwidth of both passbands increased slightly. However, because of the strong reflection of the signal voltage at each rod transmission line terminal, the fluctuation amplitude of the voltage transmission coefficient in the passband with frequency became more severe, and the shape of the passband became worse. A large fluctuation in the voltage transmission coefficient will cause serious distortion of the signal transmission, and this means the frequency characteristics of the channel will become worse. When $R_{2}$ reaches $10^{10}$ (nearly branch off), the voltage transmission coefficient in the two passbands of the channel only increased ten times, but the frequency characteristics of the channel were seriously deteriorated due to the sharp fluctuation of the voltage transmission coefficient in the passband. Therefore, to ensure that the channel has good frequency characteristics, the value of $R_{2}$ should not be greater than $1000 \Omega$.

3.2. Analysis of the Reflection Coefficient Phase. According to equation (7), the terminal reflection coefficient of the transmission line can improve the signal voltage transmission coefficient of the transmission line under the condition that the reflection coefficient phase is less than $120^{\circ}$, so as to improve the voltage transmission capacity of the wired drill pipe channel. It can be seen from Figure 6 that the center frequency of the second passband is approximately 11.7 MHz. At this frequency, the voltage transmission capacity of the $i$-th transmission line of the rod in the channel is simulated, and the phase of the reflection coefficient at the terminal of the transmission line is calculated. Figure 7 is the circuit diagram of a two-series wired drill pipe, and the circuit was simulated using EDA software (NI Multisim 14.0). In the diagram, the frequency of the signal source is 11.7 MHz, the voltage amplitude of the signal source $V_{1}=1 \mathrm{~V}, R_{2}=1000 \Omega, C_{2}=171 \mathrm{pF}, \mathrm{T} 1$ and $\mathrm{T} 2$ are inductive couplers, and $\mathrm{W} 1$ and $\mathrm{W} 2$ are the transmission lines of the rod. As the resistance of impedance matching compensation element $R_{2} \neq 137 \Omega$, the transmission line terminals are in different degrees of impedance mismatch. XMM1 is the terminal voltage of the transmission line of the first rod, $\mathrm{XMM} 2$ is the input voltage of the transmission line of the second rod, and XMM3 is the terminal voltage of the transmission line of the second rod. Based on simulation results, the voltage transmission coefficient of the transmission line of the first rod is $\left|\eta_{d 1}\right|=\mathrm{XMM} 1 / V 1=1.26$, and the voltage transmission coefficient of the transmission line of the second rod is $\left|\eta_{d 2}\right|=(\mathrm{XMM} 3 / \mathrm{XMM} 2)=1.18$. Thus, $\left|\eta_{d 1}\right|$ and $\left|\eta_{d 2}\right|$ are both greater than one, which shows that the impedance mismatch of the transmission line terminal generates the reflected signal voltage. The superposition of the reflected signal and the transmitted signal at the transmission line terminal enhanced the amplitude of the transmitted signal. The reflection coefficient of the $i$-th wired drill pipe can be calculated using $\Gamma_{i}=\left(Z_{L i}^{\prime}-R_{\mathrm{c}}\right) /\left(Z_{L i}^{\prime}+R_{\mathrm{c}}\right)$. The voltage transmission coefficients of transmission lines of each rod in the multi-combined wired drill pipe channel were simulated using a circuit simulation, as shown in Figure 7. Table 1 shows the simulation and calculation results of the voltage transmission coefficient of the transmission line of the rod and the phase values of the reflection coefficient at the terminal of the transmission line in a tenseries wired drill pipe channel.

It can be seen from Table 1 that the phase values of the reflection coefficient at each transmission line terminal are less than $120^{\circ}$, and the voltage transmission coefficient of the transmission line is greater than one, which indicates that the circuit simulation results are consistent with the theoretical analysis results. In addition, the reflected signal generated at each transmission line terminal plays an important role in improving the channel voltage transmission capacity. Figure 8 shows the influence of frequency on the terminal reflection coefficient phase of the transmission line of the last rod of the wired drill pipes when $C_{2}=171 \mathrm{pF}$ and $R_{2}=1000 \Omega$.

It can be seen from Figure 8 that the phase values of the reflection coefficient are less than $120^{\circ}$ in the passband of the transmission characteristics of the channel, which indicates that the passband was formed by the enhancement of the signal transmission due to the reflected voltage of the transmission line. In addition, when the number of wired drill pipes was small, the phase value of the reflection coefficient in the passband had a large amplitude of periodic change corresponding to the strong periodic fluctuation of the voltage transmission coefficient in the passband. The causes of channel distortion are then verified and explained. 


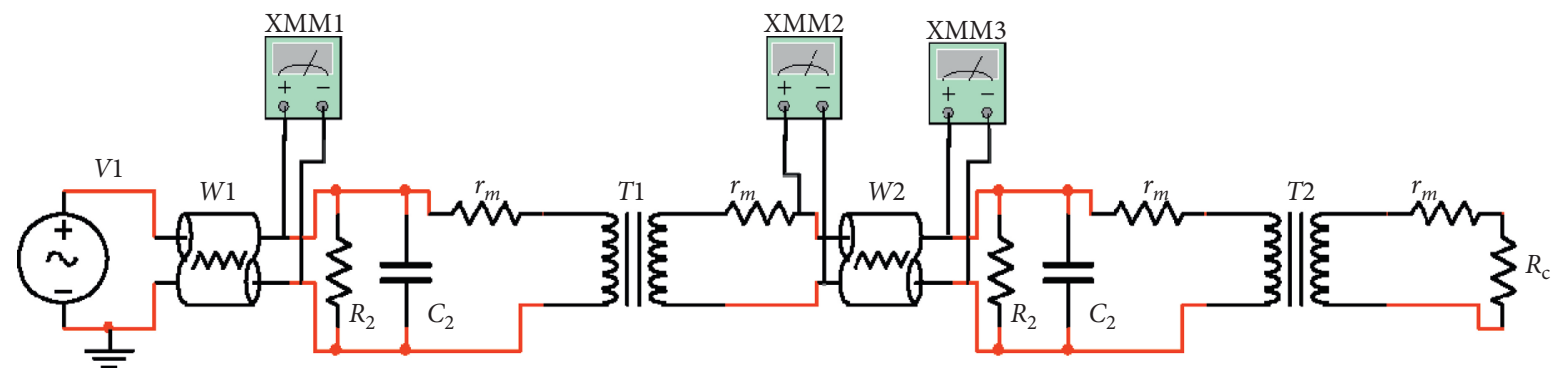

FIgURE 7: Schematic diagram of the circuit simulation for the wired drill pipe channel.

TABLE 1: The voltage transmission coefficient and terminal reflection coefficient phase of each transmission line of the rod in the channel.

\begin{tabular}{lcccccccccc}
\hline The $i$-th transmission line of the rod & 1 & 2 & 3 & 4 & 5 & 6 & 7 & 8 & 9 & 10 \\
\hline Voltage transmission coefficient & 1.33 & 1.14 & 1.24 & 1.27 & 1.17 & 1.33 & 1.14 & 1.32 & 1.15 & 1.29 \\
Reflection coefficient phase $\left({ }^{\circ}\right)$ & 28.8 & 84.4 & 17.6 & 69.4 & 11.6 & 56.8 & 13.8 & 46.4 & 25.2 & 38.2 \\
\hline
\end{tabular}

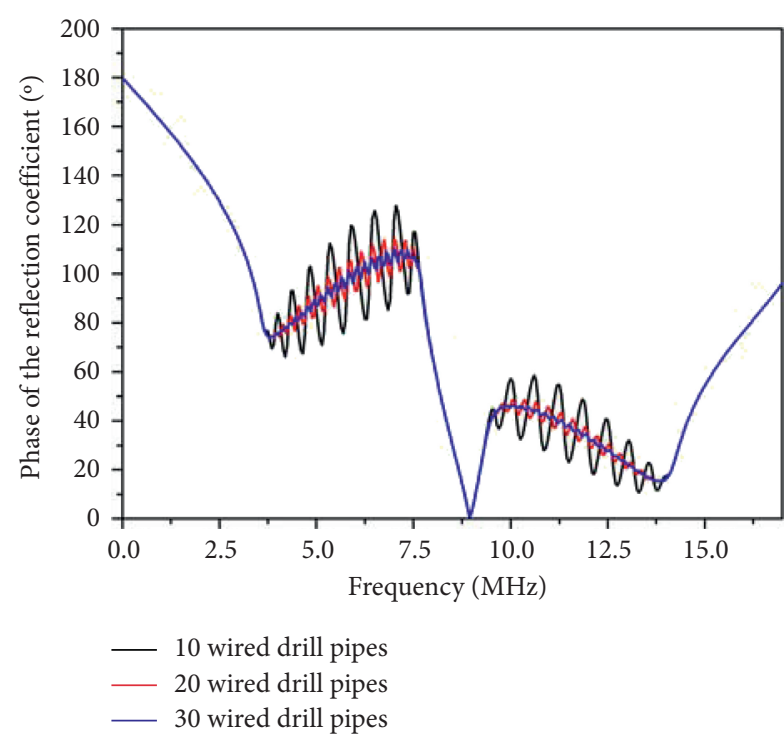

FIGURE 8: Influence of frequency on the reflection coefficient phase.

The calculation results showed that the peak voltage transfer coefficient of the channel composed of 66 drill pipes was $-18.7 \mathrm{db}$, and the channel bandwidth was $2.1 \mathrm{MHz}$ when using the second passband of Figure $6(\mathrm{~b})$ as the channel under the condition of $C_{2}=171 \mathrm{pF}$ and $R_{2}=1000 \Omega$. If the data were modulated using the QPSK method with 11.7 MHz as the carrier frequency, the data transmission rate was $2.1 \mathrm{Mbit} / \mathrm{s}$, and the relay distance was approximately $693 \mathrm{~m}$. The above calculation results showed that the relay distance would be greatly extended with the same voltage signal transmission coefficient and slightly higher data transmission rate compared to the IntelliServ network system of Grant Prideco Company [16]. Thus, the relay distance was much longer than the length of the channel formed by 30 drill pipes. All the symbol significance is shown in the Table 2.
TABLE 2: Symbol significance.

\begin{tabular}{|c|c|c|c|}
\hline Symbol & Meaning & Value & Unit \\
\hline$f_{\mathrm{d}}$ & Design frequency & 10 & $\mathrm{MHz}$ \\
\hline$\omega_{\mathrm{d}}$ & Design angular frequency & $20 \pi$ & $\mathrm{rad} / \mathrm{s}$ \\
\hline$l$ & $\begin{array}{l}\text { Length of the rod of the wired drill } \\
\text { pipe }\end{array}$ & 10.36 & $\mathrm{~m}$ \\
\hline$\hat{C}$ & $\begin{array}{l}\text { Capacitance per unit length of the } \\
\text { transmission line }\end{array}$ & $6.435 \times 10^{-11}$ & $\mathrm{~F} / \mathrm{m}$ \\
\hline$\hat{L}$ & $\begin{array}{l}\text { Inductance per unit length of the } \\
\text { transmission line }\end{array}$ & $3.798 \times 10^{-7}$ & $\mathrm{H} / \mathrm{m}$ \\
\hline$\hat{R}$ & $\begin{array}{l}\text { Resistance per unit length of the } \\
\text { transmission line }\end{array}$ & 0.0797 & $\Omega / m$ \\
\hline$\hat{G}$ & $\begin{array}{l}\text { Conductivity per unit length of } \\
\text { the transmission line }\end{array}$ & $1.351 \times 10^{-16}$ & $\mathrm{~S} / \mathrm{m}$ \\
\hline$R_{\mathrm{c}}$ & $\begin{array}{l}\text { Characteristic impedance of the } \\
\text { transmission line }\end{array}$ & 76.8 & $\Omega$ \\
\hline$k$ & Coupling coefficient & 0.8 & \\
\hline$L$ & Coil inductance & 2.3 & $\mu H$ \\
\hline$d_{0}$ & Diameter of the coil conductor & $7.5 \times 10^{-4}$ & $\mathrm{~m}$ \\
\hline$R_{2}$ & $\begin{array}{l}\text { Resistance of the impedance } \\
\text { matching compensation element }\end{array}$ & 137 & $\Omega$ \\
\hline$C_{2}$ & $\begin{array}{l}\text { Capacitance of the impedance } \\
\text { matching compensation element }\end{array}$ & 171 & $\mathrm{pF}$ \\
\hline$d_{1}$ & Inner diameter of ferrite & 0.124 & $\mathrm{~m}$ \\
\hline$d_{2}$ & Outer diameter of ferrite & 0.146 & $\mathrm{~m}$ \\
\hline$d_{c}$ & Diameter of the coil & 0.135 & $\mathrm{~m}$ \\
\hline$r_{\mathrm{m}}$ & Resistance of the coil & 0.017 & $\Omega$ \\
\hline$\mu_{\mathrm{r}}$ & $\begin{array}{c}\text { Relative permeability of the ferrite } \\
\text { material }\end{array}$ & 149 & \\
\hline$\rho$ & $\begin{array}{l}\text { Conductivity of the coil } \\
\text { conductor }\end{array}$ & $1.75 \times 10^{-8}$ & $\Omega \cdot \mathrm{m}$ \\
\hline$\gamma$ & $\begin{array}{l}\text { Propagation constant of the } \\
\text { transmission line }\end{array}$ & & \\
\hline$\eta_{d i}$ & $\begin{array}{l}\text { Voltage transmission coefficient } \\
\text { of the } i \text {-th coaxial cable }\end{array}$ & & \\
\hline$\eta_{\mathrm{ci}}$ & $\begin{array}{l}\text { Voltage transmission coefficient } \\
\text { of the } i \text {-th inductive coupler }\end{array}$ & & \\
\hline$\eta_{i}$ & $\begin{array}{l}\text { Voltage transmission coefficient } \\
\text { of the } i \text {-th wired drill pipe }\end{array}$ & & \\
\hline$\Gamma$ & $\begin{array}{l}\text { Reflection coefficient of the } \\
\text { transmission line terminal }\end{array}$ & & \\
\hline
\end{tabular}




\section{Conclusion}

(1) By considering the coaxial cable embedded in the rod of the wired drill pipe as the transmission line with the distributed parameters and the inductive coupler as the transmission line load, the transmission characteristic of the rod of the wired drill pipe was studied using transmission line theory. This theory better reflects the influence of the channel on the transmission of high-frequency signals.

(2) The determination of the electromagnetic parameters of the wired drill pipe was key to improving the transmission capability of the channel. Appropriate impedance compensation elements were connected in parallel at the terminal of the coaxial cable or the input of the inductive coupler, and the electromagnetic parameters were determined according to the impedance matching of the transmission line of the rod and the inductive coupler, which provided a basic condition for improving the transmission characteristics of the channel by adjusting the parameters of the impedance compensating elements.

(3) On the basis of the determined electromagnetic parameters of the wired drill pipe, the transmission line was in an appropriate impedance mismatch by increasing the resistance of the impedance compensating elements. The signal amplitude of the transmission line was enhanced by the reflected voltage generated by the impedance mismatch, which greatly extended the relay distance (Appendix).

\section{Appendix}

\section{For the Single-Turn Coil}

As Figure 9 shows the circumstances when the current on the single-turn coil is $I$, the positive direction of the current is the counterclockwise direction of the coil, and the radius of the coil is $r_{0}$. According to the right-hand helix rule, the magnetic field direction of any point in the coil plane is vertical and outward, which is marked as $\vec{k}$. According to the Biot-Savart law, the magnetic induction at a distance $r$ from the center of the coil generated by the current unit on the coil is

$$
d \vec{B}=\frac{\mu_{0}}{4 \pi} \frac{I d l \times \overrightarrow{r^{\prime}}}{(\overrightarrow{r l})^{3}}
$$

As $d l=r_{0} d \varphi$,

$$
d \vec{B}=\frac{\mu_{0}}{4 \pi} \cdot \frac{I r_{0} \sin \beta d \varphi}{\left(\overrightarrow{r^{\prime}}\right)^{3}} .
$$

According to the mathematical theorem of a triangle, $\sin \beta=\cos \alpha, r^{\prime} / \sin \varphi=r / \sin \alpha$, and $\left(r^{\prime}\right)^{2}=r_{0}^{2}+r^{2}-$ $2 r_{0} r \cos \varphi$. (A.2) can be derived as

$$
\overrightarrow{B(r)}=\frac{\mu_{0} I r_{0}}{4 \pi} \int_{0}^{2 \pi} \frac{\sqrt{r_{0}^{2}+r^{2} \cos ^{2} \varphi-2 r_{0} r \cos \varphi}}{\left(\sqrt{r_{0}^{2}+r^{2}-2 r_{0} r \cos \varphi}\right)^{3}} \mathrm{~d} \varphi \vec{k}
$$

Then, the magnetic flux of the unit circle, $\mathrm{d} s$, at the distance, $r$, from the center of the coil can be expressed as

$$
\begin{aligned}
\Phi & =\int \vec{B} \mathrm{~d} s=\int_{0}^{r_{0}} \vec{B}(r) \cdot 2 \pi r \mathrm{~d} r \\
& =\frac{\mu_{0} I_{0}}{2} \int_{0}^{r_{0}} \int_{0}^{2 \pi} \frac{\sqrt{r_{0}^{2}+r^{2} \cos ^{2} \varphi-2 r_{0} r \cos \varphi}}{\left(\sqrt{r_{0}^{2}+r^{2}-2 r_{0} r \cos \varphi}\right)^{3}} r \mathrm{~d} r \mathrm{~d} \varphi .
\end{aligned}
$$

So, the inductance of a single-turn coil is

$$
L=\frac{\Phi}{I}=\frac{\mu_{0} I_{0}}{2} \int_{0}^{r_{0}} \int_{0}^{2 \pi} \frac{\sqrt{r_{0}^{2}+r^{2} \cos ^{2} \varphi-2 r_{0} r \cos \varphi}}{\left(\sqrt{r_{0}^{2}+r^{2}-2 r_{0} r \cos \varphi}\right)^{3}} r \mathrm{~d} r \mathrm{~d} \varphi .
$$

For the single-turn coil embedded in a ferrite groove, as shown in Figure 4, the inductance can be expressed as

$$
\begin{aligned}
L= & \frac{\mu_{0} I_{c}}{4} \int_{0}^{\left(d_{1} / 2\right)} \int_{0}^{2 \pi} \frac{\sqrt{\left(d_{c} / 2\right)^{2}+r^{2} \cos ^{2} \varphi-d_{c} r \cos \varphi}}{\left(\sqrt{\left(d_{c} / 2\right)^{2}+r^{2}-d_{c} r \cos \varphi}\right)^{3}} r \mathrm{~d} r \mathrm{~d} \varphi \\
& +\frac{\mu d_{c}}{4} \int_{\left(d_{1} / 2\right)}^{\left(d_{2} / 2\right)} \int_{0}^{2 \pi} \frac{\sqrt{\left(d_{c} / 2\right)^{2}+r^{2} \cos ^{2} \varphi-d_{c} r \cos \varphi}}{\left(\sqrt{\left(d_{c} / 2\right)^{2}+r^{2}-d_{c} r \cos \varphi}\right)^{3}} r \mathrm{~d} r \mathrm{~d} \varphi .
\end{aligned}
$$

Because the permeability of ferrite is much higher than the permeability, thus

$$
L=\frac{\mu d_{c}}{4} \int_{\left(d_{1} / 2\right)}^{\left(d_{2} / 2\right)} \int_{0}^{2 \pi} \frac{\sqrt{\left(d_{c} / 2\right)^{2}+r^{2} \cos ^{2} \varphi-d_{c} r \cos \varphi}}{\left(\sqrt{\left(d_{c} / 2\right)^{2}+r^{2}-d_{c} r \cos \varphi}\right)^{3}} r \mathrm{~d} r \mathrm{~d} \varphi .
$$

Therefore, the permeability of ferrite under the condition of designed inductance can be designed as 


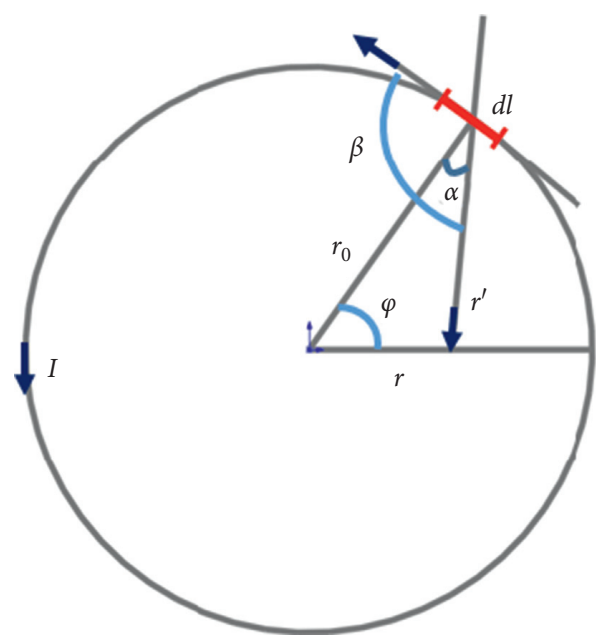

Figure 9: The single-turn coil.

$$
\mu=\frac{L}{d_{c} \int_{\left(d_{1} / 2\right)}^{\left(d_{2} / 2\right)} \int_{0}^{2 \pi}\left(\left(\sqrt{d_{c}^{2}+4 r^{2} \cos ^{2} \varphi-4 d_{c} r \cos \varphi}\right) /\left(\left(\sqrt{d_{c}^{2}+4 r^{2}-4 d_{c} r \cos \varphi}\right)^{3}\right)\right) r \mathrm{~d} r \mathrm{~d} \varphi} .
$$

The relative permeability is

$$
\mu_{r}=\frac{L}{4 \pi \times 10^{-7} d_{c} \int_{\left(d_{1} / 2\right)}^{\left(d_{2} / 2\right)} \int_{0}^{2 \pi}\left(\left(\sqrt{d_{c}^{2}+4 r^{2} \cos ^{2} \varphi-4 d_{c} r \cos \varphi}\right) /\left(\left(\sqrt{d_{c}^{2}+4 r^{2}-4 d_{c} r \cos \varphi}\right)^{3}\right)\right) r \mathrm{~d} r \mathrm{~d} \varphi} .
$$

\section{Data Availability}

The required data have been shown in tables and graphs, and there is no need to make separate tables to show the data.

\section{Conflicts of Interest}

The authors declare that they have no conflicts of interest.

\section{Acknowledgments}

This work was financially supported by the Fundamental Research Funds for the Central Universities of China (no. 27R1710013A) and the High-tech Research and Development Program of China (no. 2006AA06A101). The authors thank Accdon (http://www.accdon.com) for its linguistic assistance during the preparation of this manuscript.

\section{References}

[1] T.-S. Ma and P. Chen, "Development and use of a downhole system for measuring drilling engineering parameters," Chemistry and Technology of Fuels and Oils, vol. 51, no. 3, pp. 294-307, 2015.

[2] K. Bybee, "High-speed wired-drillstring telemetry," Journal of Petroleum Technology, vol. 60, no. 12, pp. 76-78, 2008.
[3] M. Johnson and M. Hernandez, "Along string pressure and temperature measurements in real-time: early field use and resultant value," in Proceedings of SPE/IADC Drilling Conference and Exhibition, Society of Petroleum Engineers, Amsterdam, The Netherlands, March 2009.

[4] J.-M. Godhavn, "Control requirements for automatic managed pressure drilling system," SPE Drilling \& Completion, vol. 25, no. 03, pp. 336-345, 2010.

[5] J. D. Macpherson, J. P. de Wardt, F. Florence et al., "Drillingsystems automation: current state, initiatives, and potential impact," SPE Drilling \& Completion, vol. 28, no. 04, pp. 296-308, 2013.

[6] H. Chapman, H. Anfinsen, and O. M. Aamo, "Improved kick and loss detection and attenuation in managed pressure drilling by utilizing wired drill pipe," IFAC-PapersOnLine, vol. 51, no. 8, pp. 44-49, 2018.

[7] J. E. Gravdal, M. Nikolaou, and Ø. Breyholtz, "Improved kick management during MPD by real-time pore-pressure estimation," SPE Drilling \& Completion, vol. 25, no. 04, pp. 577-584, 2010.

[8] R. Carlsen, C. Hubbell, H. D. Perez, D. S. Pixton, and A. P. Pink, "Multivariate control for managed-pressuredrilling systems by use of high-speed telemetry," SPE Journal, vol. 21, no. 02, pp. 459-470, 2016.

[9] R. A. Hedengren, C. Hubbell, H. Perez et al., "Combined rate of penetration and pressure regulation for drilling optimization by use of high-speed telemetry," SPE Drilling \& Completion, vol. 30, no. 01, pp. 17-26, 2015. 
[10] R. Hutin, R. W. Tennent, and S. V. Kashikar, "New mud pulse telemetry techniques for deepwater applications and improved real-time data capabilities," in Proceedings of SPE/ IADC Drilling Conference, p. 8, Society of Petroleum Engineers, Amsterdam, The Netherlands, February 2001.

[11] Y. Shen, Y. Su, G. Li et al., "Transmission characteristics of DPSK mud pressure signals in a straight well," Petroleum Science and Technology, vol. 29, no. 12, pp. 1249-1256, 2011.

[12] J. Schnitger and J. D. Macpherson, "Signal attenuation for electromagnetic telemetry systems," in Proceedings of SPE/ IADC Drilling Conference and Exhibition, Society of Petroleum Engineers, Amsterdam, The Netherlands, March 2009.

[13] W. Li, Z. Nie, and X. Sun, "Wireless transmission of MWD and LWD signal based on guidance of metal pipes and relay of transceivers," IEEE Transactions on Geoscience and Remote Sensing, vol. 54, no. 8, pp. 4855-4866, 2016.

[14] D. Ma, Y. Shi, and W. Zhang, "Design of acoustic transmission along drill strings for logging while drilling data based on adaptive NC-OFDM," AEU - International Journal of Electronics and Communications, vol. 83, pp. 329-338, 2018.

[15] L. Liu, W. R. Gardner, C. Robbins, and M. Memarzadeh, "Limits on data communication along the drillstring using acoustic waves," SPE Reservoir Evaluation \& Engineering, vol. 11, no. 01, pp. 141-146, 2008.

[16] L. S. Johnson, W. K. Han, Y. L. Guan, and Y. H. Lee, "Optimal energy transfer pipe arrangement for acoustic drill string telemetry," IEEE Transactions on Geoscience and Remote Sensing, vol. 52, no. 11, pp. 6999-7007, 2014.

[17] M. J. Sun, D. R. Hall, D. C. Howard et al., "Telemetry drill Pipe: enabling technology for the downhole internet," in Proceedings of SPE/IADC Drilling Conference, Society of Petroleum Engineers, Amsterdam, The Netherlands, February 2003.

[18] R. Foster and R. Macmillan, "High speed telemetry on wired drill pipe, history, and impact on drilling process," in Proceedings of Offshore Technology Conference, p. 4, Houston, TX, USA, May 2018.

[19] S. T. Edwards, C. J. Coley, N. A. Whitley et al., "A summary of wired drill pipe field trials and deployment in BP," in Proceedings of SPE/IADC Drilling Conference, p. 30, Society of Petroleum Engineers, Amsterdam, The Netherlands, March 2013.

[20] D. R. Hall, J. Fox, T. J. Wilde et al., "Transmitting data through a downhole environment," 2007.

[21] D. R. Hall and J. Fox, "Polished downhole transducer having improved signal coupling," 2003.

[22] M. Flatscher, M. Neumayer, and T. Bretterklieber, "Impedance matched electrical capacitance tomography system: front-end design and system analysis," Measurement Science and Technology, vol. 30, no. 10, 2019.

[23] J. Fadeyi, E. D. Markus, and K. Kanzumba, "A review of the impact of reflection of transient signals on tem transmission lines," in Proceedings of 2018 Open Innovations Conference, C. Ouma, Ed., IEEE, Johannesburg, South Africa, pp. 26-33, October 2018

[24] W.-D. Guo, F.-N. Tsai, G.-H. Shiue et al., "Reflection enhanced compensation of lossy traces for best eye-diagram improvement using high-impedance mismatch," IEEE Transactions on Advanced Packaging, vol. 31, no. 3, pp. 619626, 2008.

[25] T. Ma, J. Zou, P. Chen et al., "Investigation on the influence coupling drilling fluid and formation boundary on acoustic wave propagation in drill string," Geomechanics and Geophysics for Geo-Energy and Geo-Resources, vol. 6, no. 2, 2020. 